\title{
Chaos in a double pendulum
}

Troy Shinbrot

Institute for Physical Science and Technology and Department of Physics, University of Maryland, College Park, Maryland 20742

Celso Grebogi

Laboratory for Plasma Research, University of Maryland and Institute for Physical Science and

Technology and Department of Mathematics, University of Maryland, College Park, Maryland 20742

Jack Wisdom

Department of Earth and Atmospheric Sciences, Massachusetts Institute of Technology, Cambridge, Massachusetts 02139

James A. Yorke

Institute for Physical Science and Technology and Department of Mathematics, University of Maryland, College Park, Maryland 20742

A novel demonstration of chaos in the double pendulum is discussed. Experiments to evaluate the sensitive dependence on initial conditions of the motion of the double pendulum are described. For typical initial conditions, the proposed experiment exhibits a growth of uncertainties which is exponential with exponent $\lambda=7.5 \pm 1.5 \mathrm{~s}^{-1}$. Numerical simulations performed on an idealized model give good agreement, with the value $\lambda=7.9 \pm 0.4 \mathrm{~s}^{-1}$. The exponents are positive, as expected for a chaotic system.

\section{INTRODUCTION}

Sensitive dependence on initial conditions is the hallmark of chaos. ${ }^{1}$ This means that tiny separations, $\Delta x\left(t_{0}\right)$, between nearby initial conditions are amplified exponentially in time $t$ :

$$
\Delta x(t) \sim \Delta x\left(t_{0}\right) e^{\lambda t},
$$

where $\Delta x(t)$ is the separation ${ }^{2}$ between nearby trajectories and $\lambda$ is some positive constant. The double pendulum provides a simple yet dramatic demonstration of this growth. A double pendulum ${ }^{3}$ is made from two pendula attached end to end as shown in Fig. 1. Despite its simplicity, it exhibits extremely wild and unpredictable $e^{4}$ behavior. In this study we demonstrate that the underlying cause of this behavior, namely the exponential growth given in Eq. (1), can be experimentally and numerically evaluated for the double pendulum.

We begin in Sec. II by describing a simple and direct qualitative demonstration of chaos in the double pendulum. Then, in Sec. III, we discuss the root cause of the observed behavior. In the following section we describe a quantitative experiment which can be used to evaluate the rate of exponential growth, and in Sec. $V$ we discuss results obtained from the experiment. Finally, we draw conclusions.

\section{THE TANDEM DOUBLE PENDULUM}

A dramatic demonstration of the growth of separations in initial conditions intrinsic to chaotic motion can be produced by putting two identical ${ }^{5}$ double pendula in tandem, as sketched in Fig. 2.

If the pendula are released from the same small initial angle, they will oscillate in phase for long periods of time. In Fig. 3, we show actual photographs of our pendula just before release and $30 \mathrm{~s}$ after release. The pendula trajectories have stayed together for this entire time. The motion of the pendula is predictable and periodic.
If the pendula are released from a larger initial angle, however, the trajectories quickly diverge from one another. This is demonstrated in Fig. 4, where we show photographs of the pendula just before release from a large initial angle and $2 \mathrm{~s}$ after release. Clearly, the pendula trajectories have moved far apart. The motion here is unpredictable and chaotic.

We stress that these results are intrinsic to the chaotic nature of the double pendulum and are not due to imperfections ${ }^{6}$ in the double pendula or to particulars of the mechanisms of releasing the pendula. Even if the pendula were exactly identical, we could not release the pendula with the infinite precision necessary to disregard Eq. (1). Any difference in initial conditions, however tiny, is sufficient to give rise to the growth in separations shown in Fig. 4.

We also remark that sturdy construction of the tandem pendula base effectively isolates the double pendula from one another. ${ }^{7}$ The behavior observed can be fully discussed

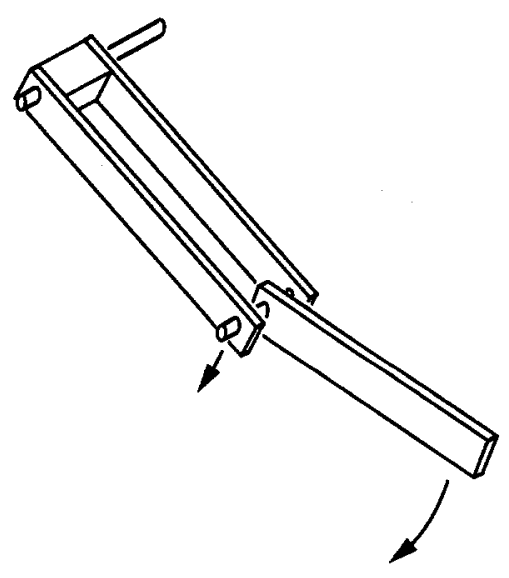

Fig. 1. A double pendulum. 


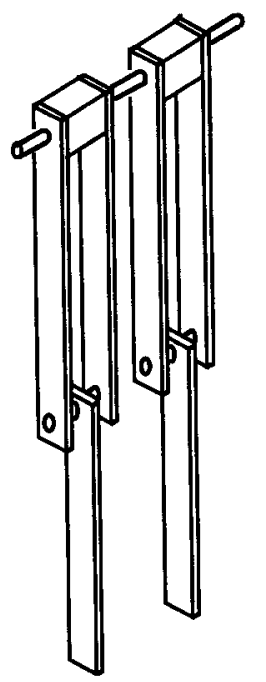

Fig. 2. Tandem double pendula.

assuming that no coupling exists between the two double pendula.

\section{CAUSE OF EXPONENTIAL GROWTH}

The growth in separations just described can occur in many extremely simple systems. For example, consider the well-known case of the logistic equation. Imagine that we want to study the ecology of a population of lions and gazelles living on a particular prairie. Let $L_{n}$ be the fraction of lions in the $n$th generation. Thus if there were 10 lions and 9990 gazelles in the first generation, $L_{1}$ would be $1 / 1000$. Now if $L_{n}$ is small, the lions will have plenty of gazelles to feast upon, and their population will grow. We can therefore write that

$$
L_{n+1}=\widehat{G} L_{n} \text {, }
$$

where $\widehat{G}$ is some constant which defines the growth rate; say $\widehat{G}=4$. Thus the number of lions will quadruple every generation in the presence of a plentiful food supply. Once $L_{n}$ becomes large, however, the number of gazelles becomes limited, and the lion population can no longer grow at its prior rate. We can easily include this in our model by making the growth rate $\widehat{G}$ decrease as the lion population grows. The simplest such model would be to write $G=G\left(1-L_{n}\right)$, where $G$ is some new constant; again say $G=4$. Thus our final model is

$$
L_{n+1}=G\left(1-L_{n}\right) L_{n} \text {. }
$$

This is known as the logistic equation. Although quite simple, it exhibits features of a chaotic system. If we take our prior starting point, $L_{1}=1 / 1000$, and look at populations after successive generations, we obtain the pattern shown in Fig. 5. While $L_{n}$ remains small, the population nearly quadruples every generation, but once $L_{n}$ becomes substantial, the behavior becomes extremely erratic and unpredictable. This is because in the logistic model, like the double pendulum, small separations grow exponentially in time.

To observe this growth, let us suppose that the initial gazelle population were 9991 instead of 9990 . This tiny, $0.01 \%$, discrepancy in $L_{1}$ would grow with every generation as shown in Fig. 6, until about ten generations, when

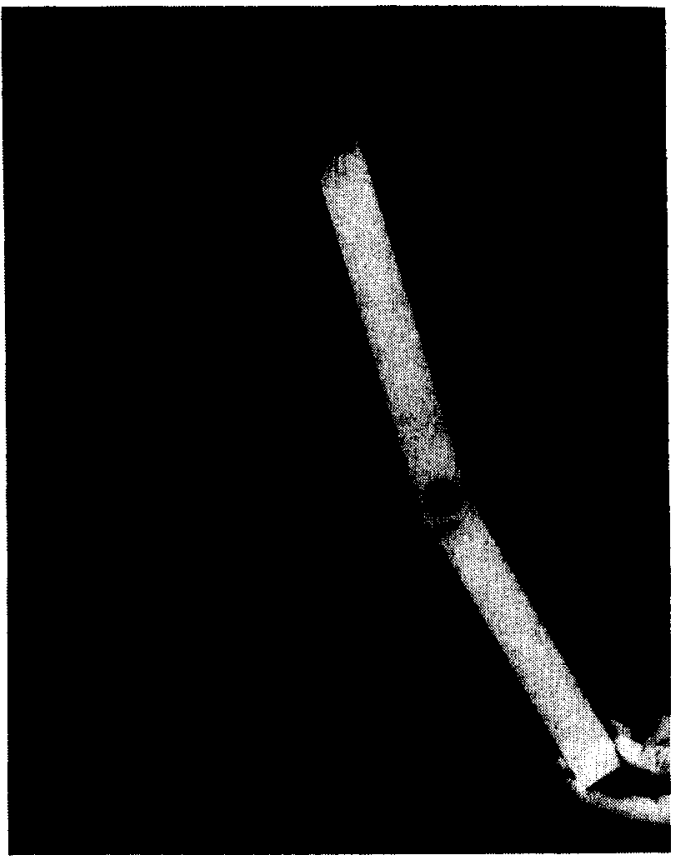

(a)

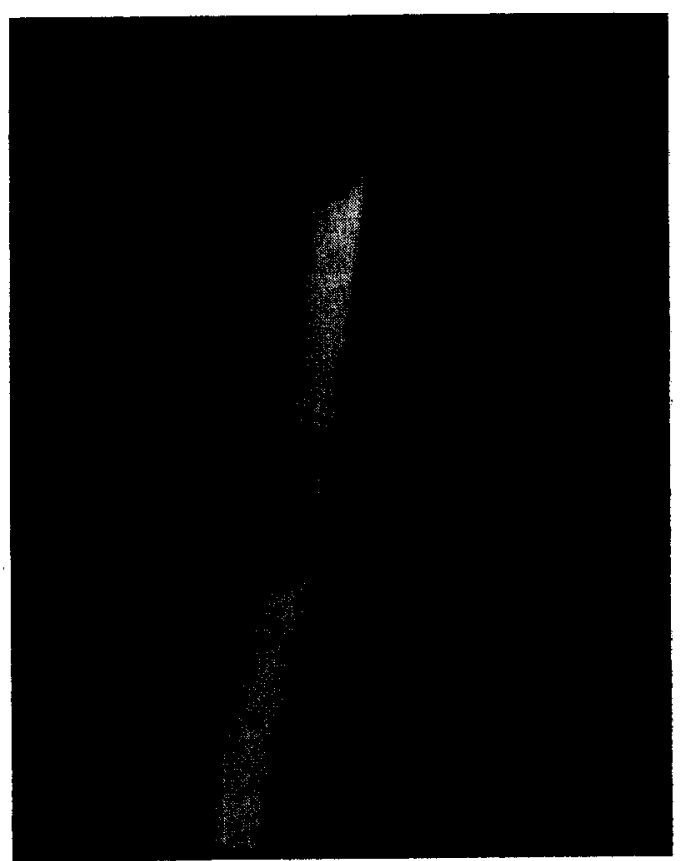

(b)

Fig. 3. Small angle motion: (a) release from small initial angle; (b) positions 30-s later.

the discrepancy would become $100 \%$. That is, if we are certain of our initial conditions to within $0.01 \%$, we can predict the outcome for only ten generations. After that point, the system will be completely unpredictable. Notice that Fig. 6 is plotted on a semilog scale. Since the plot is roughly linear on this scale, we know that growth must be roughly exponential in time on average, i.e.,

$$
\Delta L_{n}=\Delta L_{1} e^{\lambda n}
$$

where $\lambda \cong 1.35$ for Fig. 6 . This means that if we wanted to double the time for which our system remains predictable, we would have to square the initial accuracy. Since our initial accuracy in $L_{1}$ was 1 part in 10,000 , to maintain 


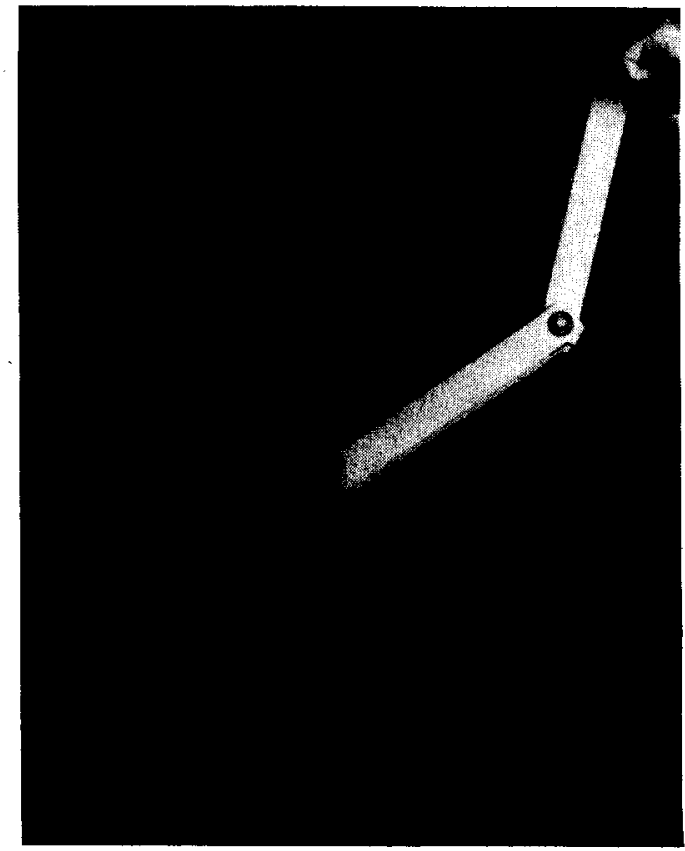

(a)

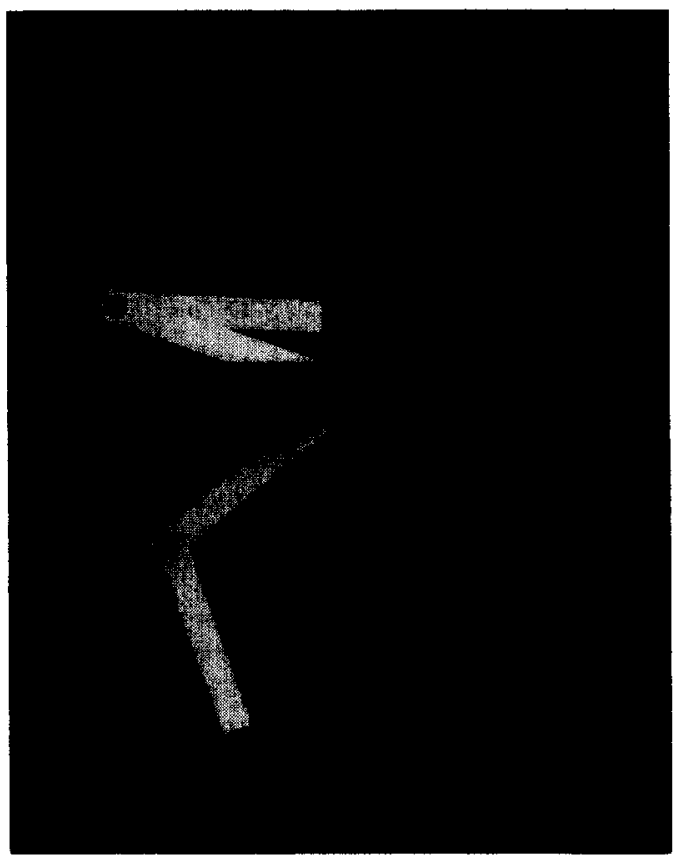

(b)

Fig. 4. Large angle motion: (a) release from large initial angle; (b) positions 2-s later.

predictability for 20 generations, we would require 1 part in $100,000,000$ accuracy, for 40 generations we would require 1 part in $10,000,000,000,000,000$ accuracy, and so forth. It is evident that no amount of initial accuracy will be sufficient to predict the outcome for long times. This is the reason that exponential growth leads to unpredictable behavior.

The origin of this exponential growth in separation between trajectories in continuous systems, like the double pendulum, can be understood in the following way. Suppose that the state of one double pendulum at time $t_{0}$ is defined by the vector $\mathbf{X}_{1}$, which would contain four components: two angles and two angular speeds. Likewise, let

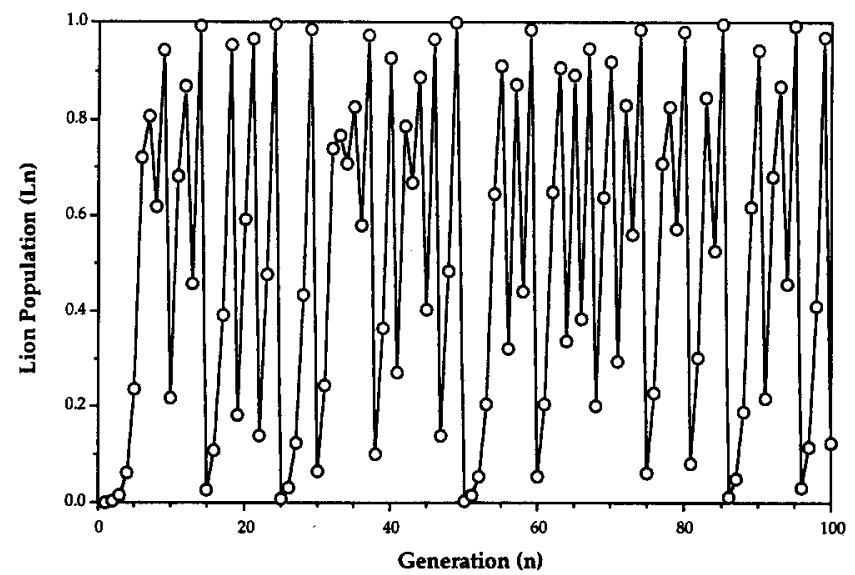

Fig. 5. Number of lions predicted in successive generations.

the state of another double pendulum-or the same pendulum during another trial-be $X_{2}$. We can expand the equations of motion in powers of $\Delta \mathbf{X}=\mathbf{X}_{1}-\mathrm{X}_{2}$ to give

$$
\Delta \stackrel{\mathrm{X}}{=} \mathbf{A}_{1} \Delta \mathbf{X}+O\left(|\Delta \mathbf{X}|^{2}\right)
$$

where $A_{1}$ is some matrix. If we start the two double pendula off at very close to the same initial condition, then $|\Delta \mathbf{X}|$ will be small, and we can neglect higher-order terms, leaving

$$
\Delta \dot{\mathbf{X}}=\mathbf{A}_{1} \Delta \mathbf{X} \text {. }
$$

The eigenvectors $\mathbf{a}_{i}$ of Eq. (6) adhere to

$$
\mathbf{a}_{i}(t)=\mathbf{a}_{i}\left(t_{0}\right) e^{\lambda_{i} t},
$$

where $i=1$ to 4 .

If the largest $\lambda_{i}$, denoted $\lambda$, is positive, we say that the motion is unstable, since fluctuations grow rapidly with time and move the two pendula away from one another. On the other hand if $\lambda$ is negative, fluctuations will rapidly vanish, and we say that the motion is stable, and the two pendula will stay nearly in synch. Stable motion is insensitive to fluctuations and tends to be predictable; conversely unstable motion tends to be unpredictable.

A good working definition of a chaos is the following. A system is said to be chaotic if the eigenvalue $\lambda$-called the Lyapunov exponent-is positive for typical initial conditions. Such a system exhibits exponential growth of distur-

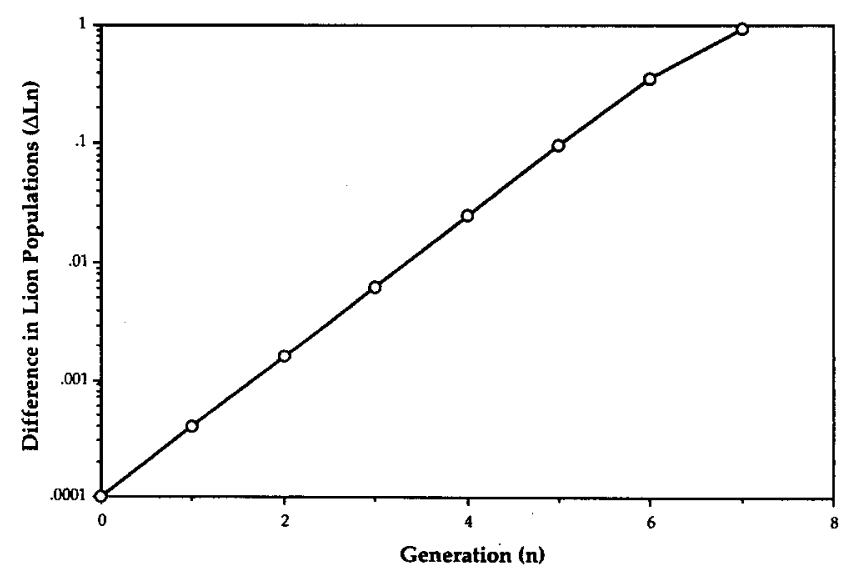

Fig. 6. Exponential growth in separations in predicted number of lions. 
bances. It should be remarked that a displacement $\Delta \mathbf{X}$ typically has components in the directions of all of the $a_{i}$, yet as time passes, the growth in the direction with the largest positive exponent will quickly swamp the growth in other directions. This largest exponent thus dominates the exponential rate of growth, so we include only this exponent in Eq. (1). Local variations ${ }^{9}$ in the Lyapunov exponent are to be anticipated, but we expect to see (1) govern the long time behavior of the system.

Here it is important to stress that the Lyapunov exponent can be expected to depend on the state of the systemi.e., the positions and speeds of the two pendula. ${ }^{10}$ Thus the exponent can locally be either negative or positive. In a chaotic system, disturbances will continually grow despite local variations. In nonchaotic systems, by contrast, disturbances can locally grow, but on average they will remain fixed. This distinction is well illustrated by considering a single, ordinary pendulum. It is easily proved ${ }^{11}$ that a single pendulum cannot be chaotic. Yet if the pendulum is released from two nearby initial conditions, both nearly vertical, the trajectories will at first quickly separate. A short time later, however, they will converge, completely compensating for the earlier separation. It is a straightforward and worthwhile exercise for the student to verify by numerical experiment ${ }^{12}$ that the time averaged exponential rate of separation for a single pendulum is zero, while for a double pendulum (released from a large initial angle), it is positive.

For small initial angles of the double pendulum, on the other hand, approximations can be made which make the equations of motion nearly linear. ${ }^{13}$ In that case, the motion becomes (nearly) predictable. The physical reason for this is that for small angles, the pendula oscillate about the rest position. This oscillation is stable and consequently predictable.

\section{MEASUREMENT OF EXPONENT}

\section{A. Physical experiment}

To analyze chaos quantitatively, just one double pendulum is needed. In this case, we release the double pendulum from, as nearly as possible, the same initial condition several times. We then measure, by a simple technique that we will describe shortly, the separation between these several

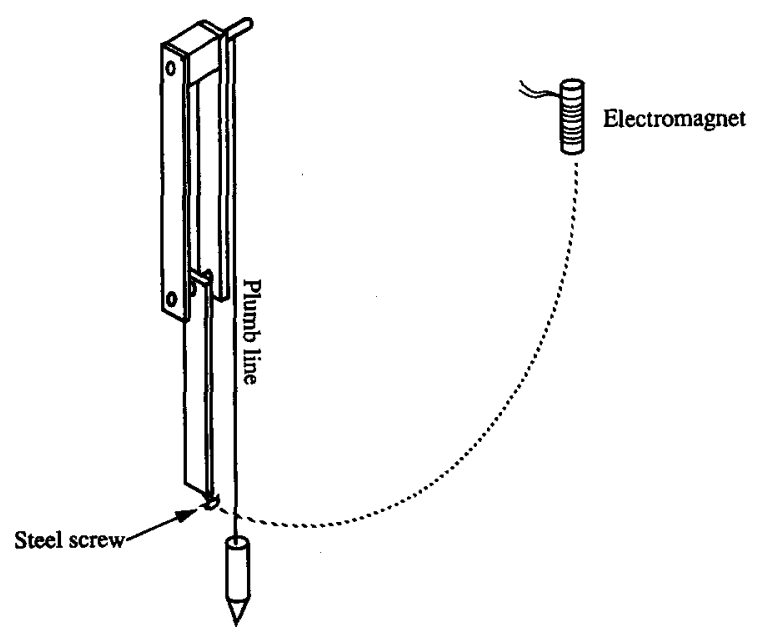

Fig. 7. Apparatus.

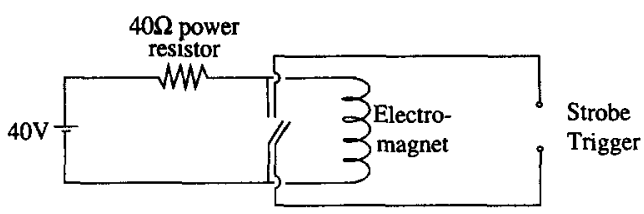

Fig. 8. Electrical schematic.

trajectories as time elapses. By plotting these separations as a function of time, we derive the exponent defined in Eq. (1).

A double pendulum ${ }^{14}$ was constructed as shown in Fig. 7 and clamped to a solid bench to ensure isolation from unwanted influences. The room was then darkened and illumination was provided with an externally triggered strobe light set to flash 25 times per second. The background behind the scene and the pendulum were painted flat black and $2 \mathrm{~mm}$ wide $\mathrm{e}^{15}$ white stripes were taped along the exposed pendula sides to provide visibility. A Polaroid $^{(}$camera was used to photograph the stroboscopic trajectory of the double pendulum. Small parallax errors were minimized by centering the camera on the scene.

A white plumb line was suspended from the upper axle of the pendulum. The plumb line gives a reference angle needed to accurately measure the angles of each pendulum. The pendulum was held in its initial position by an electromagnet which attracted the steel screw shown in the figure. The electromagnet was clamped to a laboratory stand, fixing its position in space. By means of a double throw switch, the electromagnet released the pendulum at the same time that the strobe was triggered. An electrical schematic is shown in Fig. 8.

The completed photographs were mounted on a drafting table and the angles of each pendulum were measured, aligning the plumb line to be exactly vertical. A drafting machine ${ }^{16}$ accurate to within $0.2^{\circ}$ was used to measure each successive angular position. Taking the grain of the photograph into account, an estimated final accuracy of $1^{\circ}$ was obtained.

\section{B. Numerical experiment}

In addition to the physical experiment, a numerical simulation was performed in which the equations of motion ${ }^{17}$ were integrated by the fourth order Runge-Kutta-Nystrom method. ${ }^{18}$ To ensure correspondence between physical and numerical experiments, the moments of inertia of the numerical double pendulum were set equal to those of the physical double pendulum. The latter were calculated after measuring the appropriate masses and lengths. Noise and dissipation ${ }^{19}$ were neglected in the numerical experiment.

\section{RESULTS}

Typical results ${ }^{20}$ of several experimental trials, each consisting of releasing the double pendulum as nearly as possible from the same initial position, are shown in Fig. 9. The initial position was chosen to have large pendula angles and so to exhibit chaotic motion. In the figure we plot the Euclidean distance in $\left(\vartheta_{1}, \vartheta_{2}, \vartheta_{1}, \vartheta_{2}\right)$ space ${ }^{21}$ between successive points taken at the same time after release from the different trials. Also shown, as a bold line, is the result of comparing separations produced in numerical simulations ${ }^{22}$ of a double pendulum programmed to have parameters identical with the physical pendulum. 


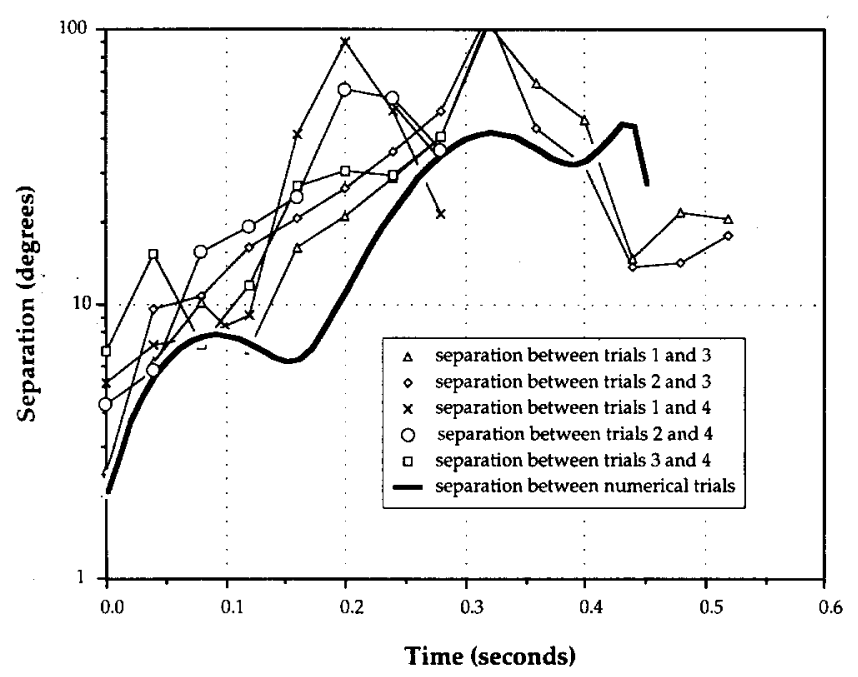

Fig. 9. Experimental results from typical initial condition; bold curve is numerical result.

In addition to the clear upward trend seen in the curves shown in Fig. 9, it is notable that a substantial undulation is present in both the physical and the numerical experiments. Earlier, we mentioned that local variations in $\lambda$ may be anticipated; here we see an illuminating example of the source of these variations. By examining the pendula trajectories, shown in Fig. 10, we see that just after being released, the outer pendulum oscillates about its vertical position. Ordinarily, this would be an unstable position, however the influence of the inner pendulum pulling the outer pendulum down is such that this position becomes stable. The sign of the Lyapunov exponents dictates stability, thus apparently during this period of time at least one of the exponents has changed from positive to negative. The occurrence of this particular change is due to the accidental choice of initial state; nevertheless it illuminates the richness of the dynamics of the double pendulum.

Further analysis was performed to compare the physical and numerical experiments. Since Fig. 9 contains large undulations, the slope of any of the curves in the figure must depend on the range of points used. Therefore it was considered prudent to create an objective criterion for the choice of points. From the definition of the Lyapunov ex-

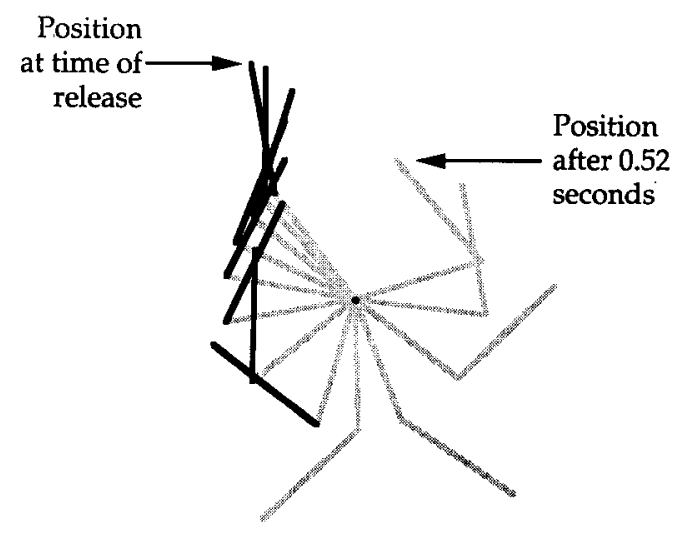

Fig. 10. Reproduction of actual stroboscopic positions of pendula (from data, first initial condition, Trial 1). Notice solid lines oscillate about vertical.
Table I. Lyapunov exponents at $\chi^{2}$, minimum.

\begin{tabular}{lcc}
\hline \hline Trials & No. points minimizing $\chi_{r}^{2}$ & Exponent \\
\hline 1 and 2 & 11 & $3.50 \pm 0.02$ \\
2 and 3 & 9 & $9.73 \pm 0.03$ \\
1 and 3 & 8 & $9.07 \pm 0.04$ \\
1 and 4 & 6 & $7.88 \pm 0.005$ \\
2 and 4 & 8 & $-0.248 \pm 0.04$ \\
3 and 4 & 8 & $6.30 \pm 0.03$ \\
1 and 5 & 4 & $4.38 \pm 0.1$ \\
2 and 5 & 6 & $12.73 \pm 0.06$ \\
3 and 5 & 7 & $12.15 \pm 0.05$ \\
4 and 5 & 7 & $12.38 \pm 0.05$ \\
\hline \hline
\end{tabular}

ponent [Eqs. (1) and (5)], the magnitude of separations between different trajectories should be as small as possible. Thus we must not discard any points near $t=0$, where the separation is smallest. To decide when to stop taking points, we adopt the following procedure. ${ }^{23}$ We examine successive numbers of points, perform a least-squares fit of the points to our expected exponential form, and calculate the reduced chi-squared for the fit. We then select the largest possible number of points which gives a minimum value of chi-squared. By this technique, we arrive at the results shown ${ }^{24}$ in Table I.

We can then calculate an average of the Lyapunov exponent, weighted in accordance to the displayed uncertainties, which gives ${ }^{25} \lambda=7.5 \pm 1.5 \mathrm{~s}^{-1}$. We can similarly compute the exponent which minimizes $\chi_{r}^{2}$ for the numerical simulation, resulting in the value $\lambda=7.9 \pm 0.4 \mathrm{~s}^{-1}$. It is worth remarking that for chaotic systems, each realization of the Lyapunov exponent is intrinsically nonreproducible, thus some uncertainty in the final estimate is unavoidable. Moreover, we must reiterate that in any real experiment, the exponential rate of separation can only be averaged over a finite time, ${ }^{26}$ while to prove that a system is chaotic, one must technically average over an infinite time span. Consequently, no experiment can definitively prove that a given system is chaotic. Nevertheless, it is fair to say that our experimental results are fully consistent with the observed chaotic behavior of the the double pendulum.

\section{CONCLUSION}

Using the double pendulum, we have successfully measured the exponential rate of separation of nearby trajectories which is characteristic of chaotic systems. The results obtained show good agreement between experiment and numerical simulation and give estimates of the Lyapunov exponent with respectable uncertainties. Most important, the Lyapunov exponent obtained by either method is unequivocally positive, as expected for a chaotic system.

The level of these experiments seems appropriate for graduate or upper level undergraduate Physics students. For more advanced students, the experiments can be extended by varying the initial energy of the pendula in a search for a transition region between nearly linear and chaotic motion. ${ }^{27}$

\section{ACKNOWLEDGMENTS}

This research was supported by the U.S. Department of Energy (Scientific Computing Staff, Office of Energy Research). 
APPENDIX A: PLANS FOR DOUBLE PENDULUM

Parts list for one pendulum

\begin{tabular}{ll}
\hline \hline Quantity & Item (all units are inches) \\
\hline 2 & $\frac{1}{4} \times 1 \frac{1}{2} \times 11$ aluminum bar stock \\
1 & $\frac{1}{2} \times 1 \frac{1}{2} \times 8 \frac{1}{2}$ aluminum bar stock \\
1 & $1 \times 1 \frac{1}{4} \times 1 \frac{1}{4}$ aluminum bar stock \\
1 & $\frac{5}{16}$ o.d. $\times 1 \frac{7}{8}$ CRS or stainless rod stock \\
1 & $\frac{5}{16}$ o.d. $\times 3 \frac{1}{2}$ CRS or stainless rod stock \\
2 & $\frac{1}{4}$ i.d. E-rings \\
1 & $1 \times 2 \times 2$ CRS bar stock \\
4 & High precision bearings (e.g., roller skate \\
1 & bearings) \\
& $\frac{3}{8}$ i.d. $\times \sim 3$ fiberglass or similar for \\
8 & sleeves (excess length allowed \\
& to fit in lathe chuck) \\
\hline \hline
\end{tabular}

Assembly:

(sleeves, E-rings, screws not shown)

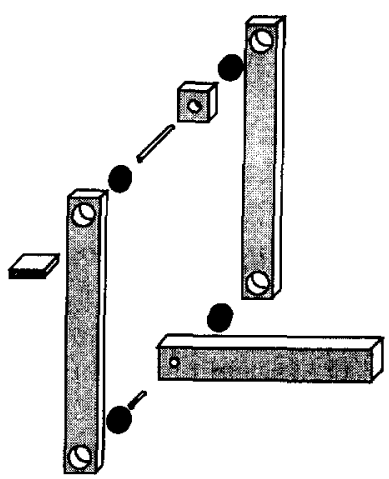

Material: Aluminum bar stock

Quantity: 1

Quantity: 2

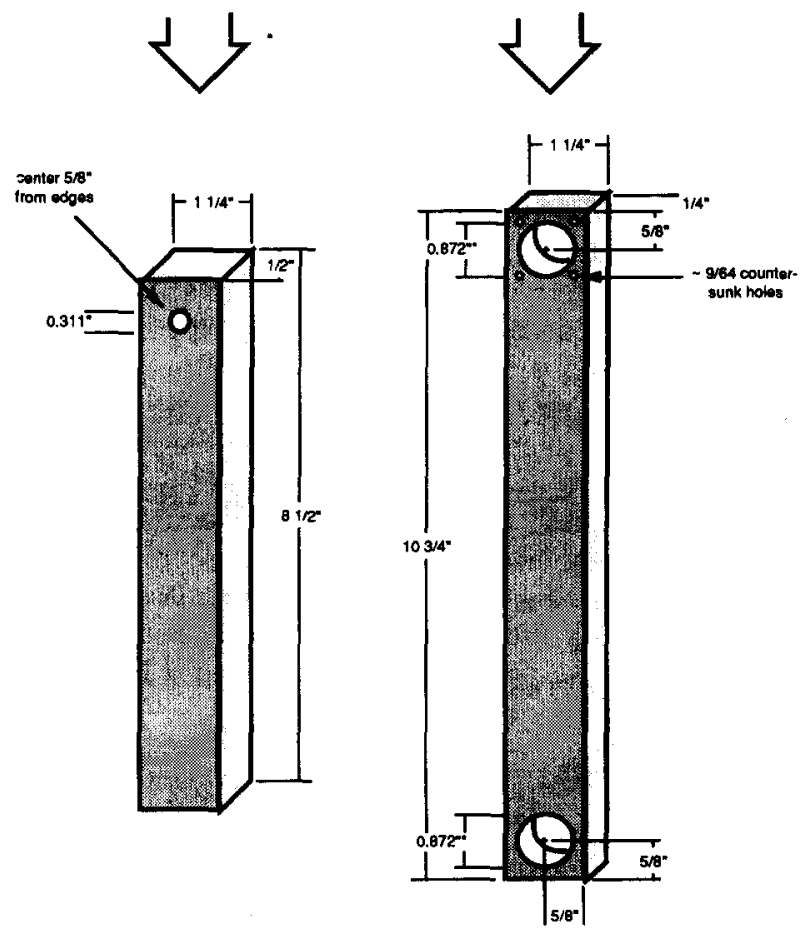

Material: Cold Rolled Steel Material: Cold Rolled Steel Quantity: 1

Quantity: 1
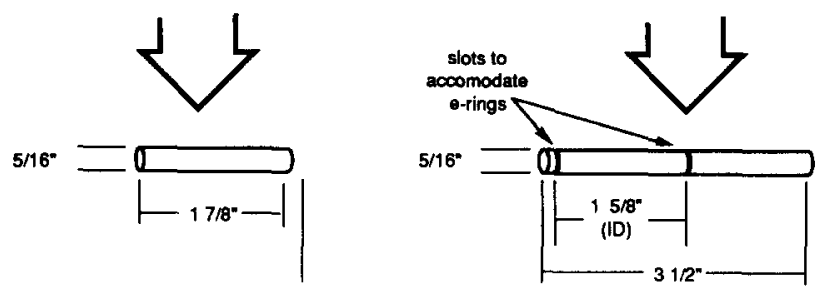

Material: Aluminum Material: Fiberglass or similar Quantity: 1 Quantity: 2
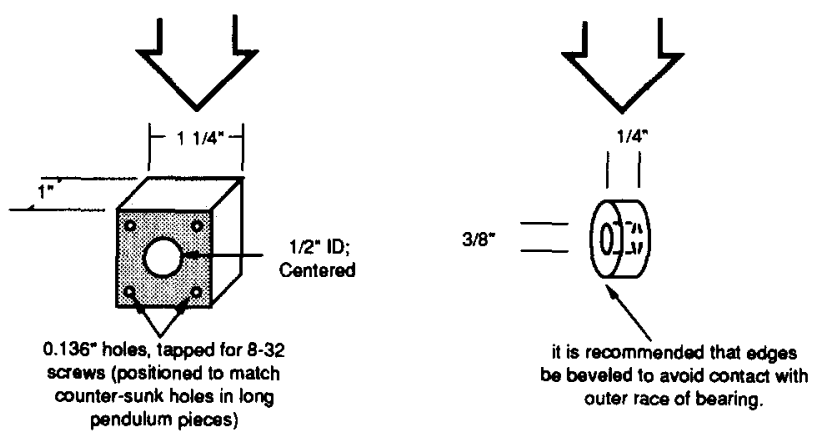

Material: Cold Rolled Steel Quantity: 1

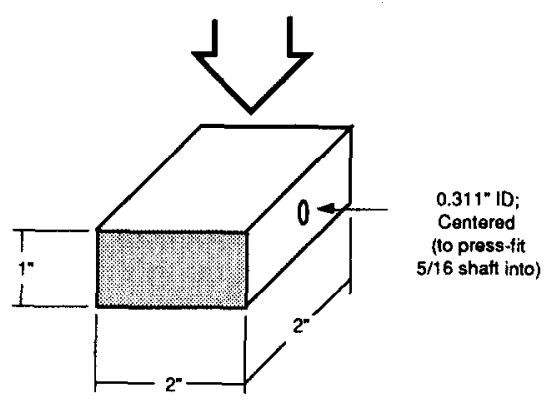

\section{APPENDIX B: EQUATIONS OF MOTION FOR DOUBLE PENDULUM}

In this appendix we derive the equations of motion for the double pendulum. We consider pendula with masses $m_{1}$ and $m_{2}$ and lengths $l_{1}$ and $l_{2}$ as shown in Fig. B1. We let $\vartheta_{1}$ and $\vartheta_{2}$ denote the angles displaced from equilibrium.

The potential energy is easily verified to be

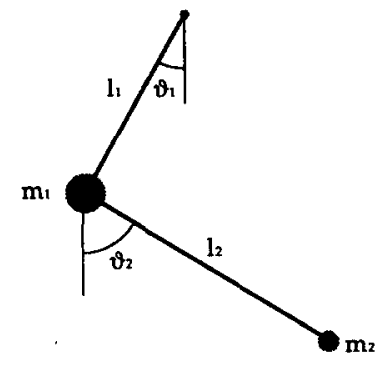

Fig. B1. Definitions. 


$$
\begin{aligned}
V= & l_{1}\left(1-\cos \vartheta_{1}\right) m_{1} g+\left[l_{1}\left(1-\cos \vartheta_{1}\right)\right. \\
& \left.+l_{2}\left(1-\cos \vartheta_{2}\right)\right] m_{2} g,
\end{aligned}
$$

while the kinetic energy is

$$
\begin{aligned}
T= & \frac{1}{2}\left(m_{1}+m_{2}\right) l_{1}^{2} \grave{\vartheta}_{1}^{2}+\frac{1}{2} m_{2} l_{2}^{2} \grave{\vartheta}_{2}^{2} \\
& +m_{2} l_{1} l_{2} \dot{\vartheta}_{1} \dot{\vartheta}_{2} \cos \left(\vartheta_{1}-\vartheta_{2}\right) .
\end{aligned}
$$

The Euler-Lagrange equations of motion then give $\cos (\Delta \vartheta) \ddot{\vartheta}_{1}+\left(l_{2} / l_{1}\right) \ddot{\vartheta}_{2}=\stackrel{\vartheta}{\vartheta}_{1}^{2} \sin (\Delta \vartheta)-\left(g / l_{1}\right) \sin \vartheta_{2}$,

$$
\begin{aligned}
& \cos (\Delta \vartheta) \ddot{\vartheta}_{2}+\left(\mu l_{1} / l_{2}\right) \ddot{\vartheta}_{1} \\
& \quad=-\dot{\vartheta}_{2}^{2} \sin (\Delta \vartheta)-\left(g \mu / l_{2}\right) \sin \vartheta_{1},
\end{aligned}
$$

where $\Delta \vartheta \equiv \vartheta_{1}-\vartheta_{2}$ and $\mu \equiv 1+\left(m_{1} / m_{2}\right)$. These equations can be separated to produce

$$
\begin{aligned}
& \ddot{\vartheta}_{1}=\frac{g\left(\sin \vartheta_{2} \cos (\Delta \vartheta)-\mu \sin \vartheta_{1}\right)-\left(l_{2} \grave{\vartheta}_{2}^{2}+l_{1} \dot{\vartheta}_{1}^{2} \cos (\Delta \vartheta)\right) \sin (\Delta \vartheta)}{l_{1}\left(\mu-\cos ^{2}(\Delta \vartheta)\right)} \\
& \ddot{\vartheta}_{2}=\frac{g \mu\left(\sin \vartheta_{1} \cos (\Delta \vartheta)-\sin \vartheta_{2}\right)+\left(\mu l_{1} \grave{\vartheta}_{1}^{2}+l_{2} \dot{\vartheta}_{2}^{2} \cos (\Delta \vartheta)\right) \sin (\Delta \vartheta)}{l_{2}\left(\mu-\cos ^{2}(\Delta \vartheta)\right)}
\end{aligned}
$$

Equations (B5) and (B6) are highly nonlinear and can only be integrated numerically, yielding a time series, or trajectory, of values for $\vartheta_{1}, \vartheta_{2}, \vartheta_{1}$, and $\vartheta_{2}$.

For small oscillations, the dynamics of the double pendulum change. In this case, we use small angle approximations for the trigonometric functions, yielding

$$
\begin{aligned}
& \ddot{\vartheta}_{1} \cong g\left(\vartheta_{2}-\mu \vartheta_{1}\right) / l_{1}(\mu-1), \\
& \ddot{\vartheta}_{2} \cong g \mu\left(\vartheta_{1}-\vartheta_{2}\right) / l_{2}(\mu-1) .
\end{aligned}
$$

These are coupled linear oscillator equations discussed in any standard classical dynamics text. The equations are linear and will therefore produce regular motion. Thus we

\begin{tabular}{|c|c|c|}
\hline & Trial 2 & \\
\hline 1 & 141.1 & 169.3 \\
\hline 2 & 137.0 & 179.1 \\
\hline 3 & 129.4 & 182.6 \\
\hline 4 & 117.4 & 190.3 \\
\hline 5 & 100.8 & 204.0 \\
\hline 6 & 97.5 & 205.6 \\
\hline 7 & 55.1 & 186.3 \\
\hline 8 & 22.7 & 140.2 \\
\hline 9 & -1.8 & 66.2 \\
\hline 10 & -1.8 & -38.0 \\
\hline 11 & -18.0 & -120.8 \\
\hline 12 & -48.1 & -182.0 \\
\hline 13 & -78.7 & -216.1 \\
\hline 14 & -103.3 & -233.2 \\
\hline \multirow[t]{2}{*}{15} & -120.0 & -235.4 \\
\hline & Trial 3 & \\
\hline 1 & 142.6 & 170.3 \\
\hline 2 & 140.0 & 179.5 \\
\hline 3 & 131.7 & 192.1 \\
\hline 4 & 120.9 & 204.0 \\
\hline 5 & 106.2 & 209.9 \\
\hline 6 & 91.6 & 196.1 \\
\hline 7 & 63.0 & 157.2 \\
\hline 8 & 30.0 & 90.9 \\
\hline 9 & -1.0 & 7.9 \\
\hline 10 & -4.2 & 0.0 \\
\hline 11 & -10.0 & -102.3 \\
\hline 12 & -38.5 & -189.1 \\
\hline 13 & -71.7 & -216.6 \\
\hline 14 & -99.9 & -245.5 \\
\hline 15 & -119.1 & -260.2 \\
\hline
\end{tabular}
expect that the exponential rate of growth of fluctuations should become vanishingly small as the initial displacements of the pendula are reduced. ${ }^{28}$

\section{APPENDIX C: DATA}

Trials following beginning from (as nearly as possible) the same initial condition. Fewer points taken from trials 4 and 5 because the first three trials revealed that minimum $\chi_{r}^{2}$ slope requires $<15$ points.

\begin{tabular}{ccc}
\hline \hline Time $\times 25(\mathrm{~s})$ & $\vartheta_{1}(\mathrm{deg})$ & $\vartheta_{2}(\mathrm{deg})$ \\
\hline & Trial 1 & \\
1 & 141.7 & 169.8 \\
2 & 136.9 & 179.6 \\
3 & 127.8 & 197.5 \\
4 & 114.8 & 202.2 \\
5 & 98.7 & 205.9 \\
6 & 78.9 & 205.0 \\
7 & 50.6 & 180.5 \\
8 & 15.7 & 127.1 \\
9 & -2.3 & 47.2 \\
10 & -2.3 & -57.2 \\
11 & -22.0 & -135.1 \\
12 & -52.8 & -189.8 \\
13 & -83.1 & -220.0 \\
14 & -106.6 & -231.2 \\
15 & -121.0 & -233.0 \\
& & \\
\hline
\end{tabular}


Trial 4

141.2

137.1

128.6

115.4

98.7

103.0

50.5

17.1

$-3.8$

$-22.3$
168.8

171.7

197.3

206.4

202.3

204.9

179.3

128.5

50.2

$-56.0$
Trial 5

141.1

139.8

132.0

175.2

197.7

$120.5 \quad 208.6$

$105.9 \quad 209.6$

$\begin{array}{ll}123.6 & 192.7\end{array}$

$22.2 \quad 146.3$

108.0

31.0

$\left(\vartheta_{1}, \vartheta_{2}, \grave{\vartheta}_{1}, \grave{\vartheta}_{2}\right)$-space separations between trials ( $\left.\mathrm{deg}\right)$

\begin{tabular}{|c|c|c|c|c|c|c|c|c|c|c|}
\hline $\begin{array}{c}\text { Time } \times \\
25(s)\end{array}$ & $\begin{array}{c}\text { Trials } \\
1 \text { and } 2\end{array}$ & $\begin{array}{c}\text { Trials } \\
2 \text { and } 3\end{array}$ & $\begin{array}{c}\text { Trials } \\
1 \text { and } 3\end{array}$ & $\begin{array}{c}\text { Trials } \\
1 \text { and } 4\end{array}$ & $\begin{array}{c}\text { Trials } \\
2 \text { and } 4\end{array}$ & $\begin{array}{c}\text { Trials } \\
3 \text { and } 4\end{array}$ & $\begin{array}{c}\text { Trials } \\
1 \text { and } 5\end{array}$ & $\begin{array}{c}\text { Trials } \\
2 \text { and } 5\end{array}$ & $\begin{array}{c}\text { Trials } \\
3 \text { and } 5\end{array}$ & $\begin{array}{l}\text { Trials } \\
4 \text { and }\end{array}$ \\
\hline 1 & 1.0 & 2.4 & 2.5 & 7.0 & 6.9 & 6.8 & 5.2 & 4.3 & 3.9 & 4.3 \\
\hline 2 & 14.5 & 9.6 & 6.2 & 11.0 & 23.3 & 15.4 & 7.1 & 5.8 & 10.8 & 5.4 \\
\hline 3 & 15.3 & 10.7 & 10.1 & 4.5 & 14.8 & 7.1 & 7.6 & 15.5 & 5.7 & 4.2 \\
\hline 4 & 15.8 & 16.3 & 6.9 & 8.9 & 24.1 & 11.8 & 9.1 & 19.4 & 6.7 & 7.8 \\
\hline 5 & 16.9 & 20.7 & 16.3 & 24.6 & 8.1 & 27.2 & 41.6 & 24.8 & 32.5 & 25.8 \\
\hline 6 & 23.9 & 26.5 & 21.2 & 34.2 & 13.1 & 30.9 & 89.3 & 60.6 & 79.9 & 58.3 \\
\hline 7 & 10.7 & 36.3 & 29.4 & 3.2 & 9.7 & 29.8 & 50.8 & 56.2 & 53.6 & 48.7 \\
\hline 8 & 17.3 & 51.1 & 41.2 & 3.9 & 14.1 & 41.3 & 21.6 & 36.5 & 33.8 & 23.9 \\
\hline 9 & 19.0 & 112.6 & 104.3 & 18.9 & 24.6 & 108.1 & & & & \\
\hline 10 & 20.1 & 44.0 & 63.7 & & & & & & & \\
\hline 11 & 16.2 & 32.6 & 47.5 & & & & & & & \\
\hline 12 & 9.9 & 13.9 & 14.9 & & & & & & & \\
\hline 13 & 8.4 & 14.2 & 21.8 & & & & & & & \\
\hline 14 & 4.5 & 18.0 & 20.9 & & & & & & & \\
\hline
\end{tabular}

${ }^{1}$ For a nice introduction to this subject, the reader is referred to $D$. Ruelle, "Strange attractors," Math. Intelligencer 2, 126-137 (1980),

${ }^{2}$ Technically, this separation is measured in phase space.

${ }^{3}$ Plans for a double pendulum appear in Appendix A.

${ }^{4}$ P. H. Richter and H. J. Scholz, "The planar double pendulum," Film C1574 (Naturwiss. Ser. 9, No. 7/C1574) Publ. Wiss. Film, Sekt. Techn. Wiss., Göttingen, 1986, and related models in P. H. Richter, "Die Theorie des Kreisels in Bildern," Report 226, Inst. Dyn. Syst., Univ. Bremen, 1990.

${ }^{5}$ The double pendula we used were made as nearly identical as possible by keeping all pairs of components clamped together during machining.

${ }^{6}$ The pendula are manifestly nearly identical, for trajectories did not separate when the pendula were released from small angles. Even modest differences would prevent the pendula from oscillating in phase for 30 or more seconds.

${ }^{7}$ This can be verified by releasing only one of the pendula from a moderate angle. Coupling would cause sympathetic oscillations-which were not observed in our apparatus-in the second, stationary, pendulum.

${ }^{8}$ Technically, the term Lyapunov exponent applies in the limit as $|\Delta \mathbf{X}| \rightarrow 0$ and $t \rightarrow \infty$. Since these limits are not realized in practical experiments, we will not make this distinction.

${ }^{9}$ These variations are ubiquitous; notice that Fig. 6 shows variations from linearity even for the simple logistic model.

${ }^{10} \mathrm{We}$ will discuss a particular instance of this phenomenon shortly.

"A good discussion appears in G. L. Baker and J. P. Gollub, Chaotic Dynamics, An Introduction (Cambridge U.P., New York, 1990), pp. 79.
${ }^{12}$ The student should be cautioned to choose initial conditions with the same energy. Failing to do this will result in a secular growth in separations which will unnecessarily complicate the problem.

${ }^{13}$ See Appendix B.

${ }^{14}$ Plans in Appendix A.

${ }^{15}$ The stripes were chosen to be the minimum thickness that would produce clear images when photographed. The strobe flash rate was determined so as to maximize the number of exposures captured for later analysis. A faster rate would produce overlap between the thin white stripes in successive exposures.

${ }^{16}$ Photographs were also digitized and angles evaluated by computer, however, the drafting machine was found to be faster and equally accurate.

${ }^{17}$ See Appendix B.

${ }^{18}$ Numerical integration for the double pendulum problem is discussed in D. R. Stump, "Solving classical mechanics problems by numerical integration of Hamilton's equations," Am. J. Phys. 54, 1096-1 100 (1986). ${ }^{19}$ This is justifiable in that dissipation could have little effect in the first $\frac{1}{2} \mathrm{~s}$ that we had to work with in the physical experiment.

${ }^{20}$ Typical results shown to reduce clutter in figure. Data from all trials appear in Appendix C.

${ }^{21}$ In our programs, conjugate momenta were computed automatically, so actual phase space distances could be calculated as well. However, the expressions for the momenta required many cumbersome calculations which necessarily reduced accuracy. For this study we chose to opt for simplicity over rigor in distance calculations.

${ }^{22}$ Several different variations from the nominal initial condition were ex- 
amined. Lyapunov exponents were virtually identical, hence only one simulation curve is shown. The separations shown differ initially by $1 \%$ in both $\vartheta_{1}$ and $\vartheta_{2}$.

${ }^{23}$ In less advanced classroom or laboratory settings, less rigorous techniques can of course be used. For example, all points $<\frac{1}{2} \mathrm{~s}$ from release can be examined. Some accuracy will be lost, but the goal of the experiment-measurement of a positive exponent-would still be achievable.

${ }^{24}$ The uncertainties shown are statistical uncertainties from least-squares fits and do not account for variations between trials.

${ }^{25}$ Uncertainty shown is standard error of weighted average.

${ }^{26}$ This is known in the literature as the problem of finite time estimation; see P. Grassberger, R. Badii, and A. Politi, "Scaling laws for invariant measures on hyperbolic and non-hyperbolic attractors," J. Stat. Phys. 51, 135-178 (1988) or E. Ott, C. Grebogi, and J. A. Yorke, "Theory of first order phase transitions for chaotic attractors of non-linear dynamical systems," Phys. Lett. A 135, 343-348 (1989).

${ }^{27}$ This has been done in our laboratory, confirming that small initial angles give approximately periodic motion with $\lambda \cong 0$, while initial angles larger than about $30^{\circ}$ give chaotic motion with $\lambda>0$.

${ }^{28}$ For a good discussion of the limitations of neglecting nonlinear terms in two oscillator systems, the reader is referred to $\mathbf{G}$. $\mathbf{H}$. Walker and $\mathbf{J}$. Ford, "Amplitude instability and ergodic behavior for conservative nonlinear oscillator systems," Phys. Rev. 188, 416-432 (1969). 